\title{
Universal skein theory for finite depth subfactor planar algebras
}

\author{
Vijay Kodiyalam and Srikanth Tupurani ${ }^{1}$
}

\begin{abstract}
We describe an explicit finite presentation for a finite depth subfactor planar algebra. We also show that such planar algebras are singly generated with the generator subject to finitely many relations.
\end{abstract}

Mathematics Subject Classification (2010). Primary 46L37; Secondary 57M99.

Keywords. Skein theory, subfactors, planar algebras.

\section{Introduction}

The main result of this paper expresses a subfactor planar algebra of finite depth as a quotient of a universal planar algebra on finitely many generators by a planar ideal generated by finitely many relations. Such a presentation is often referred to as a skein theory for the planar algebra. In addition, we also show that such a planar algebra is generated by a single element subject to finitely many relations.

Our presentation is universal in the following sense. We specify a small set of 'templates' for relations in any finite depth subfactor planar algebra. If $P$ is one such with depth at most $k$, taking a basis of $P_{k}$ to be a generating set and specialising these templates to $P$ presents it.

Skein theories for planar algebras have been the subject of several studies beginning with [8] for the group subfactor planar algebra and [4] and [6] for irreducible depth two planar algebras to the more recent [9] for the $D_{2 n}$ planar algebras, [1] for a unified treatment of the ADE planar algebras, [10] for the Haagerup subfactor planar algebra and [2] for the extended Haagerup subfactor planar algebra. One of the main results of each of these papers is a nice skein theory for a finite depth subfactor planar algebra or a family of such.

The methods of this paper do not by any means give any such nice skein theories for finite depth subfactor planar algebras. The point is to show that all such planar

\footnotetext{
${ }^{1}$ We thank V.S. Sunder for useful discussions. We also thank the referees for a very careful reading and various suggestions to improve the clarity and, in particular, for a simplification of the proof of (12) of Theorem 3.2 .
} 
algebras have a skein theory, or equivalently, a finite presentation. In particular, we make no attempt at being parsimonious with the relations.

In Section 2 we quickly recall basic definitions and properties of subfactor planar algebras. Section 3 is about certain relationships between tangles that we call templates and certain relationships between templates that we call consequences. Section 4 gives a finite presentation of a finite depth subfactor planar algebra. In Section 5 we make a couple of simple observations including the single generation of finite depth subfactor planar algebras.

\section{Subfactor planar algebras}

The purpose of this section is to fix our notations and conventions regarding planar algebras. We assume that the reader is familiar with planar algebras as in [3] or in [5] so we will be very brief.

Planar algebras are collections of vector spaces equipped with an action by the coloured operad of planar tangles. The vector spaces are indexed by the set $\mathrm{Col}=$ $\left\{0_{+}, 0_{-}, 1,2, \ldots\right\}$, whose elements are called colours. We endow this set with the partial order that restricts to the usual order on $\mathbb{N}$ and such that $0_{ \pm}$are incomparable and less than 1.

We will not define a tangle but merely note the following features. Each tangle has an external box, denoted $D_{0}$, and a (possibly empty) ordered collection of internal non-nested boxes denoted $D_{1}, D_{2}, \ldots$. Each box has an even number (again possibly 0 ) of points marked on its boundary. A box with $2 n$ points on its boundary is called an $n$-box or said to be of colour $n$. There is also given a collection of disjoint curves each of which is either closed, or joins a marked point on one of the boxes to another such. For each box having at least one marked point on its boundary, one of the regions (i.e. connected components of the complement of the boxes and curves) that impinge on its boundary is distinguished and marked with a $*$ placed near its boundary. The whole picture is to be planar and each marked point on a box must be the end-point of one of the curves. Finally, there is given a chequerboard shading of the regions such that the $*$-region of any box is shaded white. A 0 -box is said to be a $0_{+}$box if the region touching its boundary is white and a $0_{-}$box otherwise. A 0 without the \pm qualification will always refer to $0_{+}$. A tangle is said to be an $n$-tangle if its external box is of colour $n$. Tangles are defined only up to a planar isotopy preserving the $*$ 's, the shading, and the ordering of the internal boxes.

We illustrate several important tangles in Figure 1. This figure uses the following notational device introduced in [7]. A strand in a tangle with a non-negative integer, say $t$, adjacent to it will indicate a $t$-cable of that strand, i.e. a parallel cable of $t$ strands, in place of the one actually drawn.

A useful labelling convention for tangles is to decorate its tangle symbol, such as $I, E L, M$, or $T R$, with subscripts and a superscript that give the colours of its internal 


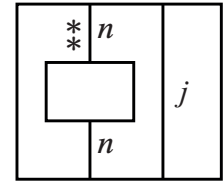

$I_{n}^{n+j}:$ Inclusion

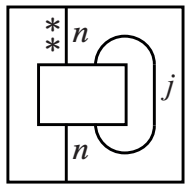

$E R_{n+j}^{n}:$ Right expectation

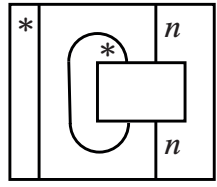

$E L_{n+1}^{n+1}:$ Left expectation

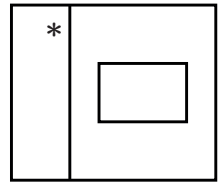

$I_{O_{-}}^{1}:$ Inclusion

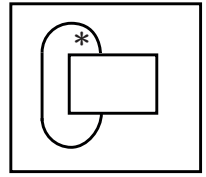

$E R_{1}^{0_{-}}$: Right expectation

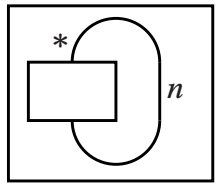

$T R_{n}^{0}:$ Trace

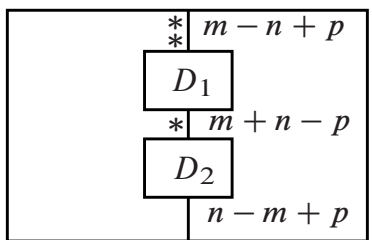

$M_{m, n}^{p}:$ Multiplication

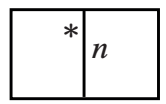

$1^{n}$ : Mult. identity

Figure 1. Some important tangles $(m, n, j \geq 0,|m-n| \leq p \leq m+n)$.

boxes and external box respectively. With this, we may dispense with showing the shading, which is then unambiguously determined.

The basic operation that one can perform on tangles is substitution of one into a box of another. If $T$ is a tangle that has some internal boxes $D_{i_{1}}, \ldots, D_{i_{j}}$ of colours $n_{i_{1}}, \ldots, n_{i_{j}}$ and if $S_{1}, \ldots, S_{j}$ are arbitrary tangles of colours $n_{i_{1}}, \ldots, n_{i_{j}}$, then we may substitute $S_{t}$ into the box $D_{i_{t}}$ of $T$ for each $t$ - such that the "*'s match" - to get a new tangle that will be denoted $T{ }^{\circ}\left(D_{i_{1}}, \ldots, D_{i_{j}}\right)\left(S_{1}, \ldots, S_{j}\right)$. The collection of tangles along with the substitution operation is called the coloured operad of planar tangles.

A planar algebra $P$ is an algebra over the coloured operad of planar tangles. By this, is meant the following: $P$ is a collection $\left\{P_{n}\right\}_{n \in \mathrm{Col}}$ of vector spaces and linear maps $Z_{T}^{P}: P_{n_{1}} \otimes P_{n_{2}} \otimes \cdots \otimes P_{n_{b}} \rightarrow P_{n_{0}}$ for each $n_{0}$-tangle $T$ with internal boxes of colours $n_{1}, n_{2}, \ldots, n_{b}$. The collection of maps is to be compatible with substitution of tangles and renumbering of internal boxes in an obvious manner. For a planar algebra $P$, each $P_{n}$ acquires the structure of an associative, unital algebra with multiplication defined using the tangle $M_{n, n}^{n}$ and unit defined to be $1_{n}=Z_{1^{n}}^{P}(1)$.

Among planar algebras, the ones that we will be interested in are the subfactor planar algebras. These are complex, finite-dimensional and connected in the sense that each $P_{n}$ is a finite-dimensional complex vector space and $P_{0_{ \pm}}$are one dimensional. They have a positive modulus $\delta$, meaning that closed loops in a tangle $T$ contribute a 
multiplicative factor of $\delta$ in $Z_{T}^{P}$. They are spherical in that for a 0 -tangle $T$, the function $Z_{T}^{P}$ is not just a planar isotopy invariant, but also an isotopy invariant of the tangle regarded as embedded on the surface of the two sphere. Further, each $P_{n}$ is a $C^{*}$-algebra in such a way that, for an $n_{0}$-tangle $T$ with internal boxes of colours $n_{1}, n_{2}, \ldots, n_{b}$ and for $x_{i} \in P_{n_{i}}$, the equality $Z_{T}^{P}\left(x_{1} \otimes \cdots \otimes x_{b}\right)^{*}=Z_{T^{*}}^{P}\left(x_{1}^{*} \otimes \cdots \otimes x_{b}^{*}\right)$ holds, where $T^{*}$ is the adjoint of the tangle $T$, which, by definition, is obtained from $T$ by reflecting it. Finally, the trace $\tau: P_{n} \rightarrow \mathbb{C}=P_{0}$ defined by

$$
\tau(x)=\delta^{-n} Z_{T R_{n}^{0}}^{P}(x)
$$

is postulated to be a faithful, positive (normalised) trace for each $n \geq 0$.

Any subfactor planar algebra $P$ (of modulus $\delta$ ) contains the distinguished Jones projections $e_{n} \in P_{n}$ for $n \geq 2$ defined by $e_{n}=\delta^{-1} Z_{E^{n}}^{P}(1)$ and their non-normalised versions $E_{n}=Z_{E^{n}}^{P}(1)$. A subfactor planar algebra $P$ is said to be of finite depth if there is a positive integer $k$ such that $P_{k+1}=P_{k} E_{k+1} P_{k}$ and the smallest such $k$ is said to be the depth of $P$.

Fix any positive integer $k$. In the rest of the paper, the tangles $T^{n}$ defined for $n \in \mathrm{Col}$ as in Figure 2 play an important role. In this and all subsequent tangle figures, we suppress drawing the external box of tangles and adopt the convention that the $*$ of the external box (if it is an $n$-box with $n>0$ ) is at the top left corner. Shaded regions of a tangle will be to the left traversing any string along the direction indicated on it. The tangle $T^{n}$ is an $n$-tangle with $n-k+1$ internal boxes for $n \geq k$
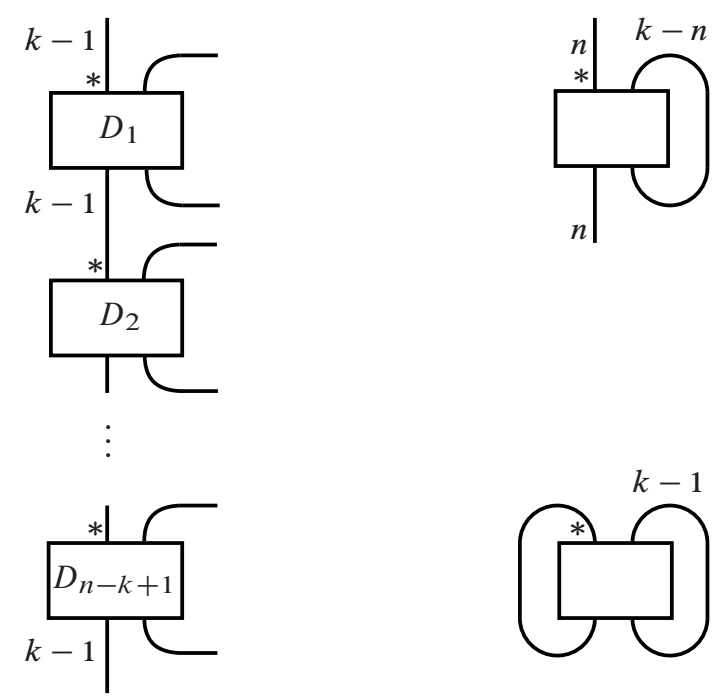

Figure 2. The tangles $T^{n}$ for $n \geq k, 0 \leq n<k$ and $n=0_{-}$.

and one internal box for $n<k$, all of colour $k$. Note that for $n<k, T^{n}=E R_{k}^{n}$ while $T^{k}=I_{k}^{k}$. 
The following proposition is well known. We only give a proof for completeness and since it is completely planar-algebraic. Note the absence of any assumptions on the planar algebra.

Proposition 2.1. Let $P$ be any planar algebra and suppose that for some positive integer $k, 1_{k+1} \in P_{k} E_{k+1} P_{k}$. For all $m, n \geq k$, there is an isomorphism of $\left(P_{k-1}-P_{k-1}\right)$-bimodules,

$$
P_{m} \otimes_{P_{k-1}} P_{n} \cong P_{m+n-(k-1)},
$$

which is implemented by the tangle $M=M_{m, n}^{m+n-(k-1)}$ (see Figure 1).

Proof. From $1_{k+1} \in P_{k} E_{k+1} P_{k}$ we see easily - see the proof of Lemma 5.7 of [4] that, for all $n \geq k, P_{n+1}=P_{k} E_{k+1} E_{k+2} \ldots E_{n+1} P_{n}$ and, inductively, $P_{n+1}=$ $P_{k} E_{k+1} E_{k+2} \ldots E_{n+1} P_{k} E_{k+1} E_{k+2} \ldots E_{n} P_{k} \ldots P_{k} E_{k+1} P_{k}$. Expressed pictorially, this yields the surjectivity of $Z_{T^{n}}^{P}$ for all $n \geq k$.

Now consider the tangle $M=M_{m, n}^{m+n-(k-1)}$. Thus $Z_{M}^{P}: P_{m} \otimes P_{n} \rightarrow P_{m+n-(k-1)}$ and a little thought shows that this is a $P_{k-1}-P_{k-1}$-bimodule map that factors through $P_{m} \otimes_{P_{k-1}} P_{n}$. The surjectivity of this map follows from the tangle equation $M{ }^{\circ}\left(D_{1}, D_{2}\right)\left(T^{m}, T^{n}\right)=T^{m+n-(k-1)}$.

The proof of injectivity uses the tangles $W=W_{n, 2 n-k+1}^{n}$ and $W^{*}$ of Figure 3 . First use the surjectivity above for $m=n$ to conclude that there exist $x_{i}, y_{i} \in P_{n}$,
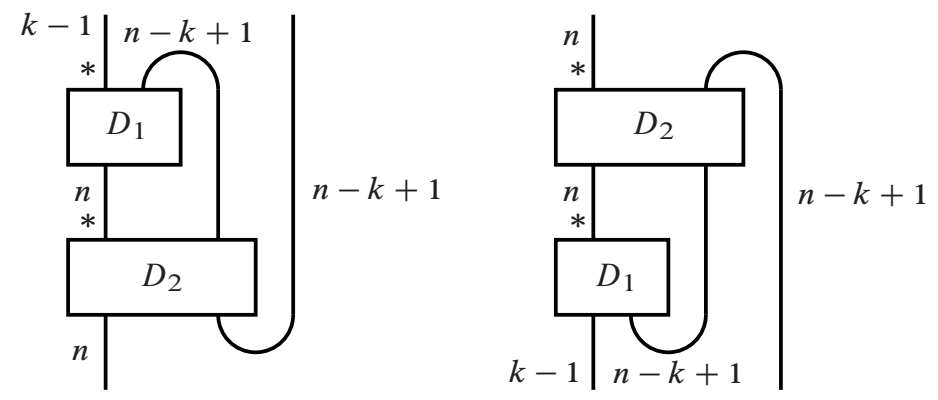

Figure 3. The tangles $W$ and $W^{*}$.

for $i \in I$, a finite set such that $1_{2 n-(k-1)}=\sum_{i \in I} Z_{M}^{P}\left(x_{i} \otimes y_{i}\right)$. Hence, for any $v \in P_{n}, Z_{W}^{P}\left(v, 1_{2 n-(k-1)}\right)=\sum_{i \in I} Z_{W{ }^{\circ} D_{2} M}^{P}\left(v \otimes x_{i} \otimes y_{i}\right)$. Equivalently, for all $v \in P_{n}$, we have $v=\sum_{i \in I} Z_{E R_{n}^{k-1}}\left(v x_{i}\right) y_{i}$.

Now, we claim that if $\sum_{j \in J} u_{j} \otimes v_{j} \in \operatorname{ker}\left(Z_{M}^{P}\right)$, then,

$$
\sum_{j \in J} u_{j} \otimes v_{j}=\left(\sum_{i \in I, j \in J} u_{j} \otimes Z_{E R_{n}^{k-1}}\left(v_{j} x_{i}\right) y_{i}\right)-\left(\sum_{i \in I, j \in J} u_{j} Z_{E R_{n}^{k-1}}\left(v_{j} x_{i}\right) \otimes y_{i}\right) .
$$


In fact, the left hand side equals the first term on the right hand side while the second term on the right vanishes since for each $i \in I$, the sum $\sum_{j \in J} u_{j} Z_{E R_{n}^{k-1}}\left(v_{j} x_{i}\right)$ is of the form $Z_{W^{*}{ }_{{ }^{\circ} D_{2} M}}^{P}\left(x_{i} \otimes \sum_{j \in J} u_{j} \otimes v_{j}\right)=Z_{W^{*}}^{P}\left(x_{i} \otimes Z_{M}^{P}\left(\sum_{j \in J} u_{j} \otimes v_{j}\right)\right)=0$.

The displayed equation expresses $\sum_{j \in J} u_{j} \otimes v_{j}$ as an element in the kernel of the natural map $P_{m} \otimes P_{n} \rightarrow P_{m} \otimes_{P_{k-1}} P_{n}$ and concludes the proof.

We will need the following corollary whose proof follows easily by induction using Proposition 2.1.

Corollary 2.2. Let $P$ be any planar algebra and suppose that for some positive integer $k, 1_{k+1} \in P_{k} E_{k+1} P_{k}$. Then, for all $n \geq k$ there is a $\left(P_{k-1}-P_{k-1}\right)$-bimodule isomorphism

$$
P_{k} \otimes_{P_{k-1}} P_{k} \otimes_{P_{k-1}} \cdots \otimes_{P_{k-1}} P_{k} \cong P_{n}
$$

(where there are $n-k+1$ terms $P_{k}$ on the left) which is implemented by the tangle $T^{n}$.

\section{Templates and consequences}

A template is an ordered pair of tangles $(S, T)$ of the same colour but will be written as a tangle implication $S \Rightarrow T$. Given any set of templates, we will be interested in their consequences which are, by definition, those that can be obtained from them using (i) reflexivity, (ii) transitivity, and (iii) composition on the outside, i.e. elements of the smallest set of templates containing the original set and such that (i) all $T \Longrightarrow$ $T$ are in the set, (ii) if $S \Longrightarrow T$ and $T \Longrightarrow V$ are in the set, so is $S \Longrightarrow V$, and (iii) if $W$ is an arbitrary $\left(n_{0} ; n_{1}, \ldots, n_{b}\right)$ tangle and $S_{i} \Longrightarrow T_{i}$ are in the set with colour $n_{i}$, then, $W \circ_{\left(D_{1}, \ldots, D_{b}\right)}\left(S_{1}, \ldots, S_{b}\right) \Longrightarrow W \circ_{\left(D_{1}, \ldots, D_{b}\right)}\left(T_{1}, \ldots, T_{b}\right)$ is also in the set.

While templates and consequences are defined without reference to planar algebras, the motivation for the definition of consequences comes from the following. Let $P$ be a planar algebra and $B \subseteq P$, i.e. $B=\bigsqcup_{n \in \mathrm{Col}} B_{n}$ where $B_{n} \subseteq P_{n}$ for all $n \in \mathrm{Col}$. Given the pair $(P, B)$, each $\left(n_{0} ; n_{1}, \ldots, n_{b}\right)$-tangle $T$ then determines a certain subspace $R_{(P, B)}(T) \subseteq P_{n_{0}}$ defined to be (i) the span of all $Z_{T}^{P}\left(x_{1} \otimes \cdots \otimes x_{b}\right)$ for $x_{i} \in B_{n_{i}}$ if $b>0$ or (ii) the span of $Z_{T}^{P}$ (1) if $b=0$. A template $S \Longrightarrow T$ is said to hold for the pair $(P, B)$ if $R_{(P, B)}(S) \subseteq R_{(P, B)}(T)$. It is now easy to see that if a set of templates holds for $(P, B)$, then so do all their consequences.

For this paper we need a particular collection of templates shown in Figure 4 which we will refer to as the basic templates. Here $k$ is a fixed positive integer. Note that Figure 4 names each of the templates, shows them as tangle implications, and in the process, defines some tangles.

We begin with a simple but very useful lemma which we will refer to later as removing loops. 

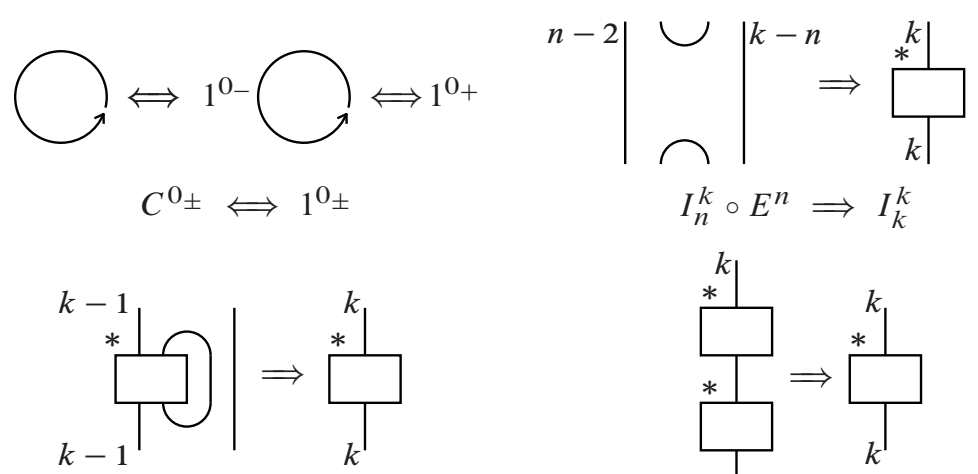

$I_{n}^{k} \circ E^{n} \Longrightarrow I_{k}^{k}$

$$
I_{k-1}^{k} \circ E R_{k}^{k-1} \Longrightarrow I_{k}^{k}
$$

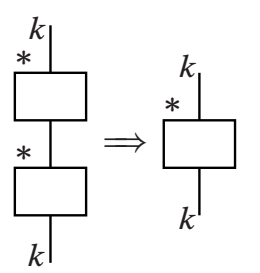

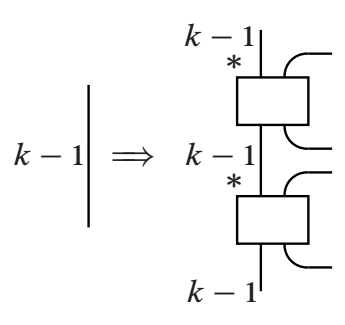

$$
M_{k, k}^{k} \Longrightarrow I_{k}^{k}
$$

$$
1^{k+1} \Longrightarrow T^{k+1}
$$

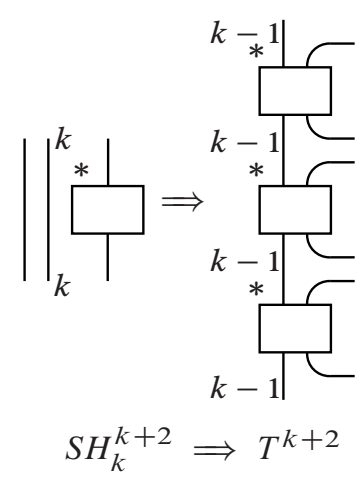

Figure 4. The basic templates: modulus, Jones projections, conditional expectation, multiplication, depth and shift ( $2 \leq n \leq k$ for the Jones projections).

Lemma 3.1. Let $S \Longrightarrow T$ be any template such that the tangle $S$ has a contractible loop somewhere in it and let $\widetilde{S}$ be $S$ with the loop removed. The modulus templates together with $S \Longrightarrow T$ have as consequence $\widetilde{S} \Longrightarrow T$.

Proof. Suppose that the contractible loop of $S$ lies in a white region. Let $W$ be the tangle obtained from $S$ by replacing the contractible loop with a $0_{+}$box numbered $b+1$, where $S$ has $b$ internal boxes. Then it is clear that $S=W{ }^{\circ} D_{b+1}\left(C^{0+}\right)$ while $\tilde{S}=W{ }^{\circ} D_{b+1}\left(1^{0_{+}}\right)$. Since the modulus tangle gives $1^{0_{+}} \Longrightarrow C^{0_{+}}$, by composing on the outside with $W$, we get $\widetilde{S} \Longrightarrow S$ and so by transitivity $\widetilde{S} \Longrightarrow T$. A similar proof applies when the loop lies in a black region.

The main result of this section is an omnibus theorem listing various consequences of the templates of Figure 4. While all the consequences are written as tangle implications, we emphasise that the proofs are purely pictorial. Recall the tangles $T^{n}$ defined for $n \in \mathrm{Col}$ in Figure 2 . 
Theorem 3.2. The following templates are all consequences of the basic templates of Figure 4.

(1) $1^{k} \Longrightarrow T^{k}$.

(2) $I_{k}^{k+1} \Longrightarrow T^{k+1}$.

(3) For all $n \in \mathrm{Col}, E R_{n+1}^{n} \circ T^{n+1} \Longrightarrow T^{n}$.

(4) For any $n \geq k, I_{n}^{n+1} \circ T^{n} \Longrightarrow T^{n+1}$.

(5) For any $n \geq k, I_{k}^{n} \Longrightarrow T^{n}$ and $1^{n} \Longrightarrow T^{n}$.

(6) For any $n \geq k, M_{n, n}^{n}{ }^{\circ}\left(D_{1}, D_{2}\right)\left(T^{n}, T^{n}\right) \Longrightarrow T^{n}$.

(7) $1^{0_{ \pm}} \Longrightarrow T^{0_{ \pm}}$and for any $n \geq 2, E^{n} \Longrightarrow T^{n}$.

(8) For any $n \geq k$ and any Temperley-Lieb tangle $Q^{n}, Q^{n} \Rightarrow T^{n}$.

(9) For any $n \geq k, S H_{n}^{n+2} \circ T^{n} \Longrightarrow T^{n+2}$.

(10) For any $n \geq 1, E L_{n}^{n} \circ T^{n} \Longrightarrow T^{n}$.

(11) For all $n \in \mathrm{Col}, I_{n}^{n+1} \circ T^{n} \Longrightarrow T^{n+1}$.

(12) For all $n \in \mathrm{Col}, M_{n, n}^{n}{ }^{\circ}\left(D_{1}, D_{2}\right)\left(T^{n}, T^{n}\right) \Longrightarrow T^{n}$.

\section{Proof.}

(1) According to the depth template $1^{k+1} \Longrightarrow T^{k+1}$. Applying $E R_{k+1}^{k}$ on both sides yields $E R_{k+1}^{k} \circ 1^{k+1} \Longrightarrow E R_{k+1}^{k} \circ T^{k+1}=M_{k, k}^{k}$. Since $E R_{k+1}^{k} \circ 1^{k+1}$ is $1^{k}$ with a contractible loop on the right, we may remove this loop by Lemma 3.1 and conclude that $1^{k} \Longrightarrow I_{k}^{k}$.

(2) Since $1^{k+1} \Longrightarrow T^{k+1}$ and $I_{k}^{k+1} \Longrightarrow I_{k}^{k+1}$, we may apply the multiplication tangle $M_{k+1, k+1}^{k+1}$ to the outside to get

$$
M_{k+1, k+1}^{k+1}{ }_{\left(D_{1}, D_{2}\right)}\left(1^{k+1}, I_{k}^{k+1}\right) \Longrightarrow M_{k+1, k+1}^{k+1}{ }_{\left(D_{1}, D_{2}\right)}\left(T^{k+1}, I_{k}^{k+1}\right) .
$$

This may also be written as $I_{k}^{k+1} \Longrightarrow T^{k+1}{ }^{\circ}{ }_{D_{2}} M_{k, k}^{k}$. Since $M_{k, k}^{k} \Longrightarrow I_{k}^{k}$, we have $T^{k+1}{ }^{\circ} D_{2} M_{k, k}^{k} \Longrightarrow T^{k+1}{ }^{\circ}{ }_{D_{2}} I_{k}^{k}=T^{k+1}$. Now appeal to transitivity.

(3) Suppose that $n<k$. Then $E R_{n+1}^{n} \circ T^{n+1}=T^{n}$, so the asserted result is clear by reflexivity. If $n \geq k$, there are two cases depending on the parity of $n-k$. These cases are shown on the left in Figure 5. We see that each is obtained by inserting a $k$-tangle into a box of $T^{n}$ and using the multiplication and conditional expectation templates, this $k$-tangle, in each case, implies $I_{k}^{k}$.

(4) Again, there are two cases according to the parity of $n-k$ which are shown on the right in Figure 5. If $n-k=2 t$, we see that $I_{n}^{n+1} \circ T^{n}=W \circ I_{k}^{k+1}$ for a suitable tangle $W$ (where $W$ has a $k+1$-box indicated by the dotted line and the rest of it looking like $\left.T^{n}\right)$. Note that, by (2), $I_{k}^{k+1} \Longrightarrow T^{k+1}$ and therefore 

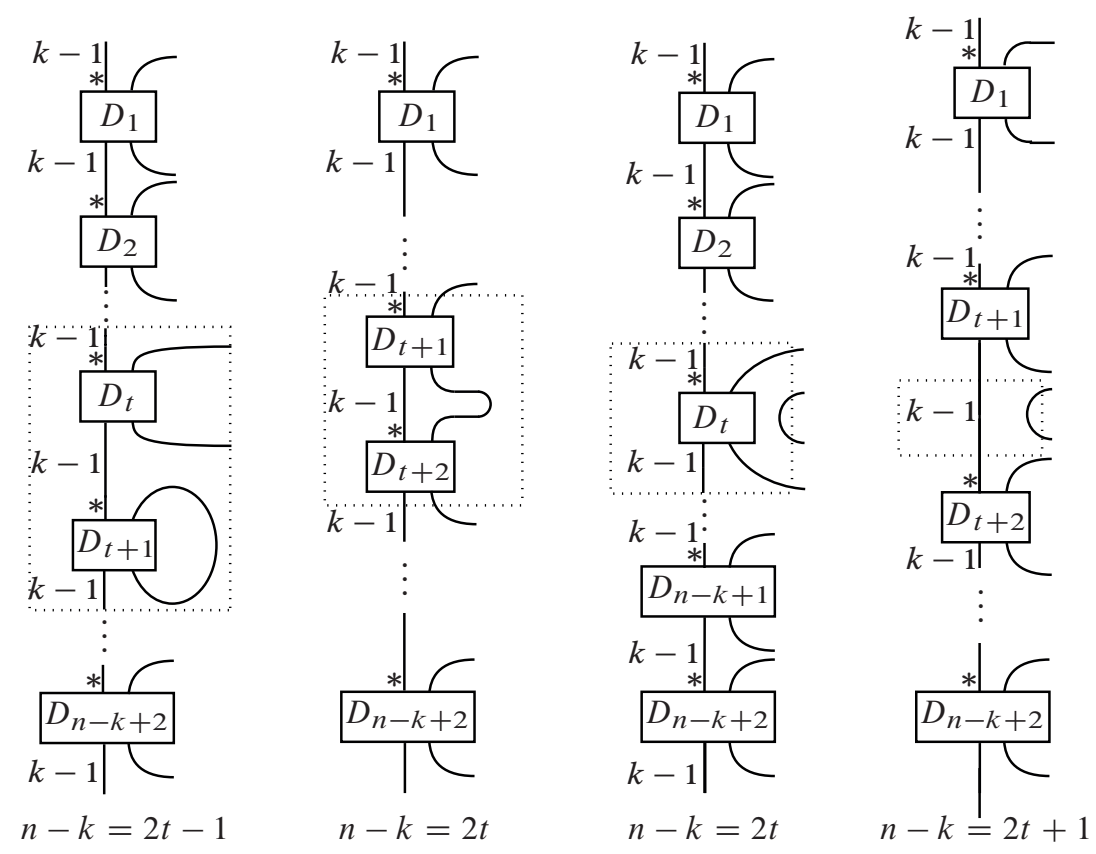

Figure 5. $E R_{n+1}^{n} \circ T^{n+1}$ and $I_{n}^{n+1} \circ T^{n}$.

$W \circ I_{k}^{k+1} \Longrightarrow W \circ T^{k+1}$. It remains only to note that $W \circ T^{k+1}=T^{n+1}$ and use transitivity to complete the proof in this case. The case $n-k=2 t+1$ is even easier. Here $I_{n}^{n+1} \circ T^{n}=T^{n+1}{ }^{\circ} D_{t+2} 1^{k}$. Since $1^{k} \Rightarrow I_{k}^{k}$, we get $I_{n}^{n+1} \circ T^{n}=T^{n+1}{ }^{\circ}{ }_{D_{t+2}} 1^{k} \Rightarrow T^{n+1}{ }^{\circ}{ }_{D_{t+2}} I_{k}^{k}=T^{n+1}$.

(5) We have by reflexivity that $I_{k}^{k} \Longrightarrow T^{k}$. Applying (4) inductively shows that for all $n \geq k, I_{k}^{n} \Longrightarrow T^{n}$. A similar proof beginning with (1) shows that $1^{n} \Longrightarrow T^{n}$.

(6) For $n=k$, this is just the multiplication template. For $n>k$, a little doodling should convince the reader that $M_{n, n}^{n}{ }^{\circ}\left(D_{1}, D_{2}\right)\left(T^{n}, T^{n}\right)=E R_{2 n-k+1}^{n} \circ T^{2 n-k+1}$. Transitivity, (3) and induction finish the proof.

(7) Begin with the identity template $1^{k} \Longrightarrow I_{k}^{k}$ and apply $E R_{k}^{0_{ \pm}}$to both sides to get $E R_{k}^{0_{ \pm}} \circ 1^{k} \Longrightarrow E R_{k}^{0_{ \pm}} \circ I_{k}^{k}=E R_{k}^{0_{ \pm}}=T^{0_{ \pm}}$. The left side of this implication is a $0^{ \pm}$-tangle which is a collection of loops which may be removed by Lemma 3.1 to yield $1^{0} \Longrightarrow T^{0_{ \pm}}$. A very similar proof beginning with the Jones projection templates gives $E^{n} \Rightarrow T^{n}$ for $2 \leq n \leq k$. To show that 
$E^{n} \Longrightarrow T^{n}$ for $n>k$, consider the following chain of implications.

$$
\begin{aligned}
E^{n} & =E R_{2 n-k-1}^{n} \circ M_{n-1, n-1}^{2 n-k-1} \circ_{\left(D_{1}, D_{2}\right)}\left(1^{n-1}, 1^{n-1}\right) \\
& \Longrightarrow E R_{2 n-k-1}^{n} \circ M_{n-1, n-1}^{2 n-k-1}{ }^{n}\left(D_{1}, D_{2}\right) \\
& \left.=E T^{n-1}, T^{n-1}\right) \\
& \Longrightarrow T_{2 n-k-1}^{n} \circ T^{2 n-k-1}
\end{aligned}
$$

where the first implication is a consequence of (5) and the second of (3) and induction.

(8) This is an easy corollary of (5), (6) and (7).

(9) Induce on $n$, with the basis case being asserted by the shift template. For $n>k$,

$$
\begin{aligned}
S H_{n}^{n+2} \circ T^{n} & =M_{n+1, k+2}^{n+2}{ }^{\left(D_{1}, D_{2}\right)}\left(S H_{n-1}^{n+1} \circ T^{n-1}, S H_{k}^{k+2}\right) \\
& \Longrightarrow M_{n+1, k+2}^{n+2}{ }^{\circ}\left(D_{1}, D_{2}\right) \\
& \left.\Longrightarrow T^{n+1}, T^{k+2}\right)
\end{aligned}
$$

where the last implication uses the multiplication and conditional expectation templates together with a suitable outside composition.

(10) First suppose that $n \geq k$. Begin with the conclusion $S H_{n}^{n+2} \circ T^{n} \Longrightarrow T^{n+2}$ in (9). Let $Q^{n+2}$ and $Q^{* n+2}$ be the Temperley-Lieb tangles shown in Figure 6, so that, by (8), $Q^{n+2} \Longrightarrow T^{n+2}$ and $Q^{* n+2} \Rightarrow T^{n+2}$. Then, with $M=$ $M_{n+2, n+2, n+2}^{n+2}$ denoting the iterated multiplication tangle, we have

$$
\begin{aligned}
M \circ\left(Q^{n+2}, S H_{n}^{n+2} \circ T^{n}, Q^{* n+2}\right) & \Longrightarrow M \circ\left(T^{n+2}, T^{n+2}, T^{n+2}\right) \\
& \Longrightarrow T^{n+2} .
\end{aligned}
$$

(For typographical convenience, we have omitted the subscripts to $\circ$ ). Hence $E R_{n+2}^{n} \circ M \circ\left(Q^{n+2}, S H_{n}^{n+2}, Q^{* n+2}\right) \Rightarrow E R_{n+2}^{n} \circ T^{n+2} \Rightarrow T^{n}$. The left hand side of this chain of implications is $E L_{n}^{n} \circ T^{n}$ with a loop at its right; therefore, using Lemma 3.1, we get the desired result. For $1 \leq n<k$, merely apply $E R_{k}^{n}$ to both sides of $E L_{k}^{k} \circ T^{k} \Longrightarrow T^{k}$.

(11) In view of (4), we only need consider the case $n<k$. If $n=0_{-}$, this is just the case $n=1$ of (10). So suppose that $0 \leq n<k$. Let $t=2 k-n+1$. Start with $I_{k}^{t} \Longrightarrow T^{t}$ deduced inductively from (4). Let $K^{t}$ and $K^{* t}$ be the TemperleyLieb tangles in Figure 6 so that, by (8), $K^{t} \Longrightarrow T^{t}$ and $K^{* t} \Rightarrow T^{t}$. Now, with $M=M_{t, t, t}^{t}, M \circ\left(K^{t}, I_{k}^{t}, K^{* t}\right) \Longrightarrow M \circ\left(T^{t}, T^{t}, T^{t}\right) \Longrightarrow T^{t}$. Applying $E R_{t}^{n+1}$ to both sides of this and removing the $k-n$ loops that arise on the left hand side, we get the desired conclusion using (3).

(12) In view of (6), we may assume that $n<k$. We first deal with the case $n \neq 0_{-}$. Let $u=2 k-n$ and $M=M_{u, u, u, u, u}^{u}$ Then, with $L^{u}$ as in Figure 6,

$$
M \circ\left(L^{u}, I_{k}^{u}, L^{u}, I_{k}^{u}, L^{u}\right) \Longrightarrow M \circ\left(T^{u}, T^{u}, T^{u}, T^{u}, T^{u}\right) \Longrightarrow T^{u} .
$$




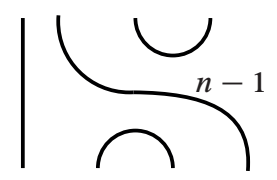

$Q^{n+2}$

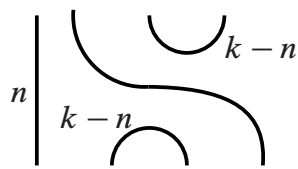

$K^{2 k-n+1}$

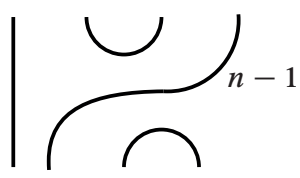

$Q^{* n+2}$

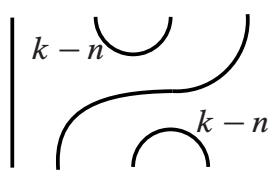

$K^{* 2 k-n+1}$

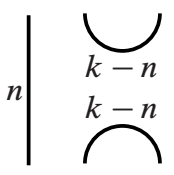

$L^{2 k-n}$

Figure 6. The tangles $Q^{n+2}, Q^{* n+2}, K^{2 k-n+1}, K^{* 2 k-n+1}$, and $L^{2 k-n}$.

As in (11), applying $E R_{u}^{n}$ to both sides and removing the $k-n$ loops gives the desired conclusion. The case $n=0_{-}$is a little more complicated. Here, let $u=2 k+1$ and $M=M_{u, u, u, u, u}^{u}$. Then, with $L^{u}, L^{* u}, W^{u}$ as in Figure 7,

$$
\begin{gathered}
M \circ\left(L^{u}, I_{k+2}^{u} \circ S H_{k}^{k+2}, W^{u}, I_{k+2}^{u} \circ S H_{k}^{k+2}, L^{* u}\right) \\
\Longrightarrow M \circ\left(T^{u}, T^{u}, T^{u}, T^{u}, T^{u}\right) \\
\Longrightarrow T^{u} .
\end{gathered}
$$

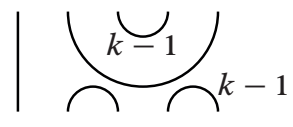

$L^{2 k+1}$

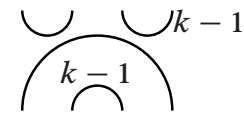

$L^{*} 2 k+1$

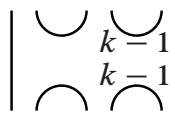

$W^{2 k+1}$

Figure 7. The tangles $L^{2 k+1}, L^{* 2 k+1}$ and $W^{2 k+1}$.

Now apply $E R_{u}^{0-}$ to both sides and remove the $k+1$ loops to get the desired conclusion. 


\section{The main theorem}

Recall from Section 3 the notion of templates holding for a pair $(P, B)$, where $P$ is a planar algebra and $B \subseteq P$. Our first observation is fairly easy to see.

Proposition 4.1. If $P$ is a subfactor planar algebra of finite depth at most $k$, and $B=B_{k}$ is a basis of $P_{k}$, then all the templates of Figure 4 hold for $(P, B)$.

Proof. The modulus templates hold for $(P, B)$ since $P$ has non-zero modulus. The Jones projections, multiplication and the conditional expectation templates hold for $(P, B)$ since their right sides are all the identity tangle $I_{k}^{k}$ and $B$ is a basis of $P_{k}$. The depth and shift templates hold since the tangles on their right, namely $T^{k+1}$ and $T^{k+2}$ surject onto their ranges by Corollary 2.2 .

Before proceeding we briefly recall (see [4] for detailed explanations) what a presentation of a planar algebra is. Given a label set $L=\bigsqcup_{n \in \mathrm{Col}} L_{n}$, there is a universal planar algebra on $L$, denoted by $P(L)$. By definition, for all $n \in \mathrm{Col}$, $P(L)_{n}$ is the vector space with basis all $L$-labelled $n$-tangles. Any subset $R \subseteq P(L)$ generates a planar ideal $I(R)$ in $P(L)$ and the corresponding quotient planar algebra is denoted $P(L, R)$.

Let $P$ be a subfactor planar algebra of depth at most $k$ and $B$ be a basis of $P_{k}$. For $b \geq 0$, let $B^{\times b}$ be the Cartesian product of $b$ copies of $B$ for $b>0$ and to be $\{1\}$ for $b=0$.

Let $L=\bigsqcup_{n \in \mathrm{Col}} L_{n}$ where the only non-empty $L_{n}$ is $L_{k}=B$. Consider the universal planar algebra $P(L)$. The templates of Figure 4 specify a subset $R \subseteq P(L)$ as follows. Fix one of the templates, say $S \Rightarrow T$, where $S$ has $b$ internal boxes and $T$ has $c$ internal boxes. Note that the colour of the internal boxes (if any) of each of $S$ and $T$ is $k$. For $\left(x_{1}, \ldots, x_{b}\right) \in B^{\times b}$ write

$$
Z_{S}^{P}\left(x_{1} \otimes \cdots \otimes x_{b}\right)=\sum_{\left\{\left(y_{1}, \ldots, y_{c}\right) \in B^{\times c}\right\}} \lambda^{\left(y_{1}, \ldots, y_{c}\right)} Z_{T}^{P}\left(y_{1} \otimes \cdots \otimes y_{c}\right),
$$

for (not necessarily unique) $\lambda^{\left(y_{1}, \ldots, y_{c}\right)} \in \mathbb{C}$ (with the obvious interpretations if $b$ or $c$ is 0$)$. This can be done since $S \Longrightarrow T$ holds for $(P, B)$. Now consider the element of $P(L)$

$$
S\left(x_{1}, \ldots, x_{b}\right)-\sum_{\left\{\left(y_{1}, \ldots, y_{c}\right) \in B^{\times c}\right\}} \lambda^{\left(y_{1}, \ldots, y_{c}\right)} T\left(y_{1}, \ldots, y_{c}\right),
$$

where $S\left(x_{1}, \ldots, x_{b}\right)$ denotes the tangle $S$ with boxes labelled $x_{1}, \ldots, x_{b}$ etc. Consider the collection consisting of one such element of $P(L)$ for each $\left(x_{1}, \ldots, x_{b}\right) \in$ $B^{\times b}$ and take the union of these collections over all templates $S \Rightarrow T$ of Figure 4 . This (clearly finite) subset of $P(L)$ is what we will call $R$. Note that $R$ is not a uniquely determined set but depends on choices. We will call this a set of relations determined by the templates of Figure 4. 
Theorem 4.1. Let $P$ be a subfactor planar algebra of finite depth at most $k$. Let $B$ be a fixed basis of $P_{k}$. Consider the labelling set $L=\bigsqcup_{n \in \mathrm{Col}} L_{n}$ where the only non-empty $L_{n}$ is $L_{k}=B$. Let $R$ be any (necessarily finite) set of relations in $P(L)$ determined by the templates in Figure 4. Then, the quotient planar algebra $P(L, R) \cong P$.

Proof. Consider the natural surjective planar algebra morphism from the universal planar algebra $P(L)$ to $P$ defined uniquely by taking a labelled $k$-box to itself regarded as an element of $P$. Equivalently, under this morphism, for any tangle $S$ all of whose internal boxes are of colour $k, S\left(x_{1}, \ldots, x_{b}\right) \mapsto Z_{S}^{P}\left(x_{1} \otimes \cdots \otimes x_{b}\right)$. Since the relations $R$ were chosen to hold in $P$, this morphism factors through the quotient planar algebra $P(L, R)$ thus yielding a surjective planar algebra morphism $P(L, R) \rightarrow P$. We wish to see that this is an isomorphism.

For $n \in \mathrm{Col}$, let $Q_{n}$ be the subspace of $P(L, R)_{n}$ spanned by all $Z_{T^{n}}^{P(L, R)}\left(x_{1} \otimes\right.$ $\left.\otimes x_{2} \otimes \cdots \otimes x_{n-k+1}\right)$ for $x_{1}, \ldots, x_{n-k+1} \in B$ if $n \geq k$ or the subspace spanned by all $Z_{T^{n}}^{P(L, R)}(x)$ for $x \in B$ if $n<k$. Let $\mathcal{T}$ be the set of all $\left(n_{0} ; n_{1}, \ldots, n_{b}\right)$ tangles $T$ such that (i) if $b>0$, then $Z_{T}\left(Q_{n_{1}} \otimes \cdots \otimes Q_{n_{b}}\right) \subseteq Q_{n_{0}}$, and (ii) if $b=0$, then $Z_{T}(1) \in Q_{n_{0}}$. Chasing definitions shows that $\mathcal{T}$ may be equivalently described as the set of $\left(n_{0} ; n_{1}, \ldots, n_{b}\right)$-tangles $T$ for which $T{ }^{\circ}\left(D_{1}, \ldots, D_{b}\right)\left(T^{n_{1}}, \ldots, T^{n_{b}}\right) \Longrightarrow T^{n_{0}}$ holds for $(P(L, R), B)$. We will show that $\mathcal{T}$ consists of all tangles, or equivalently, that $Q$ is a planar subalgebra of $P(L, R)$.

For this, we appeal to the main result of [5] which states that if $\mathcal{T}$ is a collection of tangles that is closed under composition (whenever it makes sense) and contains the tangles $1^{0_{ \pm}}, E^{n}$ for $n \geq 2, E R_{n+1}^{n}, M_{n, n}^{n}, I_{n}^{n+1}$ for all $n \in \mathrm{Col}$ and $E L_{n}^{n}$ for all $n \geq 1$, then $\mathcal{T}$ contains all tangles.

To verify the hypotheses for our $\mathcal{T}$, observe first that by definition if $T \in \mathcal{T}$ is a $\left(n_{0} ; n_{1}, \ldots, n_{b}\right)$ tangle and $S \in \mathcal{T}$ is any $n_{i}$-tangle for $i>0$, then, $T{ }^{\circ}{ }_{D_{i}} S \in \mathcal{T}$. Thus $\mathcal{T}$ is closed under composition. That the other hypotheses hold for $\mathcal{T}$ follows from the observation that the templates of Figure 4 hold for $(P(L, R), B)$ by construction of $R$ and therefore their consequences (3), (7), (10), (11), (12) of Theorem 3.2 also hold.

It follows that $Q$ is a planar subalgebra of $P(L, R)$. Since it contains all generators of $P(L, R)$, it is the whole of $P(L, R)$. In particular, $P(L, R)_{k}$ which maps onto $P_{k}$ equals $Q_{k}$ which is spanned by $B$ and so $P(L, R)_{k}$ maps isomorphically onto $P_{k}$. It easily follows that the map $P(L, R)_{n} \rightarrow P_{n}$ is an isomorphism for $n \leq k$.

For $n \geq k$, observe that Corollary 2.2 applies to $P(L, R)$ since the depth template holds for $(P(L, R), B)$. Hence we have an isomorphism of $\left(P(L, R)_{k-1}-\right.$ $\left.P(L, R)_{k-1}\right)$-bimodules

$P(L, R)_{k} \otimes_{P(L, R)_{k-1}} P(L, R)_{k} \otimes_{P(L, R)_{k-1}} \cdots \otimes_{P(L, R)_{k-1}} P(L, R)_{k} \rightarrow P(L, R)_{n}$, and therefore an isomorphism of $P_{k-1}-P_{k-1}$-bimodules

$$
P_{k} \otimes_{P_{k-1}} P_{k} \otimes_{P_{k-1}} \cdots \otimes_{P_{k-1}} P_{k} \rightarrow P(L, R)_{n} .
$$


Since the left side is, by Corollary 2.2 applied to $P$, isomorphic to $P_{n}$ while the right side maps onto $P_{n}$, it follows that $P(L, R)_{n}$ maps isomorphically to $P_{n}$ also for all $n \geq k$.

\section{On single generation}

Rather surprisingly, the fact that finite depth subfactor planar algebras are singly generated has a simple proof.

Proposition 5.1. Let $P$ be a subfactor planar algebra of finite depth at most $k$. Then $P$ is generated by a single $2 k$-box.

Proof. As a planar algebra, $P$ is generated by $P_{k}$. Since $P_{k}$ is a finite-dimensional $C^{*}$-algebra, it is singly generated, by say $x \in P_{k}$. By adding a multiple of $1_{k}$ to $x$, we may assume without loss of generality that $\tau(x) \neq 0$ (recall that $\tau(\cdot)$ is the normalised picture trace on $P$ ). Thus the planar algebra generated by $x$ and $x^{*}$ contains $P_{k}$ and must be the whole of $P$. Now consider the element $z \in P_{2 k}$ defined by Figure 8 . It should be clear that applying suitable annular tangles to $z$ yields non-zero (since $\tau(x) \neq 0$ ) multiples of $x$ and $x^{*}$. Hence the planar subalgebra of $P$ generated by $z$ contains both $x$ and $x^{*}$ and consequently is $P$.

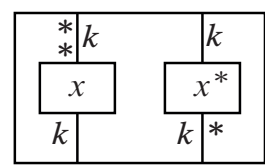

Figure 8. Definition of $z \in P_{2 k}$.

Remark 5.2. Lacking an adequate reference for the fact that finite-dimensional $C^{*}$ algebras are singly generated, we sketch a proof. For $M_{n}(\mathbb{C})$, the matrix whose only non-zero entries are 1 on the sub-diagonal is easily seen to generate it. For a direct sum of matrix algebras, take the direct sum of such elements appropriately shifted by multiples of the identity so as to make their spectra disjoint. Such an element can be shown to be a generator.

Remark 5.3. Let $d$ be a fixed positive integer. For $n \in \mathrm{Col}$, letting $P(d)_{n}$ be the vector space spanned by all $n$-tangles whose only internal boxes are of colour $d$, there is an obvious planar algebra structure on $P(d)$. What Proposition 5.1 asserts is that $P(2 k)$ maps onto any subfactor planar algebra of depth $k$.

It is natural to ask whether, when a finite depth planar algebra $P$ is presented as a quotient of $P(2 k)$ as above, the kernel is a finitely generated planar ideal. A standard proof shows that this is indeed so. 
Proposition 5.4. Let $P$ be a planar algebra and suppose that for finite label sets $L$ and $\widetilde{L}$ there are surjective planar algebra maps $\pi: P(L) \rightarrow P$ and $\tilde{\pi}: P(\widetilde{L}) \rightarrow P$. The ideal $I=\operatorname{ker}(\pi)$ is a finitely generated planar ideal of $P(L)$ if and only if $\widetilde{I}=\operatorname{ker}(\tilde{\pi})$ is a finitely generated planar ideal of $P(\tilde{L})$.

Proof. First note that universality of $P(L)$ and $P(\tilde{L})$ yield (possibly non-unique) planar algebra maps $\varphi: P(L) \rightarrow P(\widetilde{L})$ and $\widetilde{\varphi}: P(\widetilde{L}) \rightarrow P(L)$ that satisfy $\tilde{\pi} \circ \varphi=\pi$ and $\pi \circ \widetilde{\varphi}=\tilde{\pi}$.

By symmetry, it suffices to prove one implication. Suppose that $\widetilde{I}=I(\widetilde{R})$ for a finite subset $\widetilde{R} \subseteq P(\widetilde{L})$. Let $R=\widetilde{\varphi}(\widetilde{R}) \cup\{x-\tilde{\varphi} \varphi(x) \mid x \in L\}$, which is clearly a finite subset of $P(L)$. We claim that $I=I(R)$.

Clearly $R \subseteq I$ and so $I(R) \subseteq I$. The other inclusion needs a little work. First observe that $\{x-\tilde{\varphi} \varphi(x) \mid x \in L\} \subseteq R$ implies that, for all $z \in P(L), z-\tilde{\varphi} \varphi(z) \in$ $I(R)$. To see this we may reduce easily to the case that $z=T\left(x_{1}, \ldots, x_{b}\right)$ where $T$ is a $\left(n_{0} ; n_{1}, \ldots, n_{b}\right)$-tangle and $x_{i} \in L_{n_{i}}$. Then

$$
z-\tilde{\varphi} \varphi(z)=Z_{T}^{P(L)}\left(x_{1} \otimes \cdots \otimes x_{b}\right)-Z_{T}^{P(L)}\left(\tilde{\varphi} \varphi\left(x_{1}\right) \otimes \cdots \otimes \tilde{\varphi} \varphi\left(x_{b}\right)\right) .
$$

This may be expressed as a telescoping sum of $b$ terms indexed by $k=1,2, \ldots, b$ where the $k^{\text {th }}$ term is given by

$$
Z_{T}^{P(L)}\left(\tilde{\varphi} \varphi\left(x_{1}\right) \otimes \cdots \otimes \tilde{\varphi} \varphi\left(x_{k-1}\right) \otimes\left(x_{k}-\tilde{\varphi} \varphi\left(x_{k}\right)\right) \otimes x_{k+1} \otimes \cdots \otimes x_{b}\right) .
$$

Each of these terms is clearly in the planar ideal generated by $\{x-\tilde{\varphi} \varphi(x) \mid x \in L\}$ and hence in $I(R)$. Therefore $z-\tilde{\varphi} \varphi(z) \in I(R)$.

Say $z \in I$, so that $\pi(z)=0$. Then $\varphi(z) \in \operatorname{ker}(\tilde{\pi})=\widetilde{I}=I(\widetilde{R})$, i.e. $\varphi(z)$ is in the planar ideal generated by $\widetilde{R}$. It follows that $\tilde{\varphi} \varphi(z)$ is in the planar ideal generated by $\widetilde{\varphi}(\widetilde{R})$ and therefore in $I(R)$. Since $z-\tilde{\varphi} \varphi(z) \in I(R)$, we also have $z \in I(R)$ and the proof is finished.

A direct consequence of Theorem 4.1 and Propositions 5.1 and 5.4 is the following corollary.

Corollary 5.5. If $P$ is a subfactor planar algebra of finite depth at most $k$, then $P$ is generated by a single $2 k$-box subject to finitely many relations.

\section{References}

[1] S. Bigelow, Skein theory for the ADE planar algebras. J. Pure Appl. Algebra 214 (2010), 658-666. MR 2577673 Zbl 1192.46059

[2] S. Bigelow, S. Morrison, E. Peters, and N. Snyder, Constructing the extended Haagerup planar algebra. Preprint 2009. arXiv:0909.4099v1 
[3] V. F. R. Jones, Planar algebras I, New Zealand J. of Math., to appear. Preprint 1999. arXiv:math.QA/9909027

[4] V.Kodiyalam,Z.Landau, and V.S. Sunder, The planar algebra associated to a Kac algebra. Proc. Indian Acad.Sci. Math. Sci. 113 (2003), 15-51. MR 1971553 Zbl 1039.46049

[5] V. Kodiyalam and V. S. Sunder, On Jones' planar algebras. J. Knot Theory Ramifications 13 (2004), 219-247. MR 2047470 Zbl 1054.46045

[6] V. Kodiyalam and V. S. Sunder, The planar algebra of a semisimple and cosemisimple Hopf algebra. Proc. Indian Acad. Sci. Math. Sci. 116 (2006), 443-458. MR 2346882 Zbl 1116.46041

[7] V. Kodiyalam and V. S. Sunder, From subfactor planar algebras to subfactors. Internat. J. Math. 20 (2009), 1207-1231. MR 2574313 Zbl 1185.46043

[8] Z. A. Landau, Exchange relation planar algebras. Geom. Dedicata 95 (2002), 183-214. MR 1950890 Zbl 1022.46039

[9] S. Morrison, E. Peters, and N. Snyder, Skein theory for the $D_{2 n}$ planar algebras. J. Pure Appl. Algebra 214 (2010), 117-139. MR 2559686 Zbl 1191.46051

[10] E. Peters, A planar algebra construction of the Haagerup subfactor. Internat. J. Math. 21 (2010), 987-1045. MR 2679382 Zbl 05793871

Received February 23, 2010

Vijay Kodiyalam, The Institute of Mathematical Sciences, CIT Campus, Taramani, Chennai-600 113, India

E-mail: vijay@imsc.res.in

Srikanth Tupurani, The Institute of Mathematical Sciences, CIT Campus, Taramani, Chennai-600 113, India

E-mail: tsrikanth@imsc.res.in 